\title{
Utility Of 6-Azauracils in Synthesis of New Poly Heterocyclic Nitrogen Systems Bearing 1,2,4-Triazine Moiety as Antioxidant Probes
}

\author{
Hafsa Sayed and Dina A. Bakhotmah
}

\section{ABSTRACT}

\begin{abstract}
Synthesis of new poly heterocyclic nitrogen systems containing 1,2,4triazine moiety such as pyrazolines, pyrazolones, phthalazinone and 1,2,4triazinone (9-17) have been deduced from hydrazinolysis of 3,5-dichloro6,1-dihydro-1,2,4-triazine (2) which produced from the interaction between 6-azauracil (1) with $\mathrm{POCl}_{3} / \mathrm{PCl}_{5}$. Heterocyclization of 3,5-dihydrazino1,2,4-triazine (7) by reaction nitrogen / oxygen / halogen bifunctional reagents in different mediums and conditions yielded the targets 9-17 respectively. Former structure of the products has been established from their elemental analysis and spectral data (UV, IR, ${ }^{1} \mathrm{H} /{ }^{13} \mathrm{C}$ NMR and Mass). All the new targets obtained evaluated as antioxidant probes in compare with ascorbic acid in 1,1-diphenyl-2-picrylhydrazyl

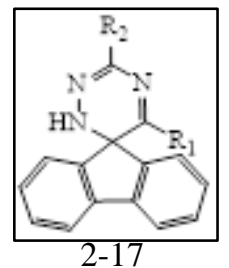
(DPPH) as a standard, were the compounds $11>10>8>12>13$ are more active probes.
\end{abstract}

Key Words: Synthesis; polyheteroaryl; 6-azauracil; antioxidant.
Published Online: December 14, 2020

ISSN: $2684-4478$

DOI :10.24018/ejchem.2020.1.6.29

\section{Hafsa Sayed}

Department of Chemistry, Faculty of Science, King Abdulaziz University, Jeddah, Saudi Arabia.

(e-mail: hafsoo_1986@hotmail.com) Dina Bakhotma*

Department of Chemistry, Faculty of Science, King Abdulaziz University, Jeddah, Saudi Arabia.

(e-mail: dbakhotmah@kau.edu.sa)

*Corresponding Author

\section{INTRODUCTION}

In recent years much attention of chemists has arisen for the synthesis, chemistry and biological activity of 6-azauracil and their derivatives as a potential chemotherapeutic agent [1] such as, antibacterial and anti-tumor [2], anti-convulsant [3], inhibitors D-amino acid oxidase [4], imaging probe for 5HT1A receptor agonist in nonhuman primates [5], [6], antimicrobial [7], and in mutations of the saccharomyces cerevisiae RPB1 gene conferring hypersensitivity to 6azauracil [8]. Most treatment of 6-azauracils with various electrophilic and/or nucleophilic reagents led to the formation of poly heterocyclic systems [9], [10], in addition produced oligonucleotides [11]-[18] (Fig. 1).

Based on these important observations, in the present study, we extended the scope of use of synthetic substituted 6-azauracils, and used to obtain 3,5-dihydrazino-1,2,4triazine which upon treated with nitrogen/ oxygen/ halogen bifunctional to give the various new poly heterocyclic nitrogen systems in view of their antioxidant activity.

\section{RESULTS AND DISCUSSION}

\section{A. Chemistry}

In view of the important work of 6-azauracils, the present work tends to obtain a various poly heterocyclic system bearing 1,2,4-triazine moiety derived from 6-azauracil (1) [19]. Thus, chlorination of spiro-5-(fluoren-9'-yl)-6-azauracil (1) by warming with $\mathrm{POCl}_{3} / \mathrm{PCl}_{5}$ along one hour, yielded 3,5-dichloro-6,1-dihydro-spiro-[5-fluoren-9'-yl]-1,2,4triazine (2).

Structure of compound $\mathbf{2}$ can be deduced from refluxing with alcohol and/or $\mathrm{H}_{2} \mathrm{O}$, afforded the corresponding asymmetrical ether $\mathbf{3}$ and/or the compound $\mathbf{1}$ (Scheme 1).

Former structure of compound $\mathbf{2}$ can be confirm from that correct elemental analysis and spectral data. IR spectrum showed a lacks of both $\mathrm{NH}, \mathrm{C}=\mathrm{O}$ absorption band, with recorded $v$ at $700 \mathrm{~cm}^{-1}$ for $\mathrm{C}-\mathrm{Cl}$ functional group.

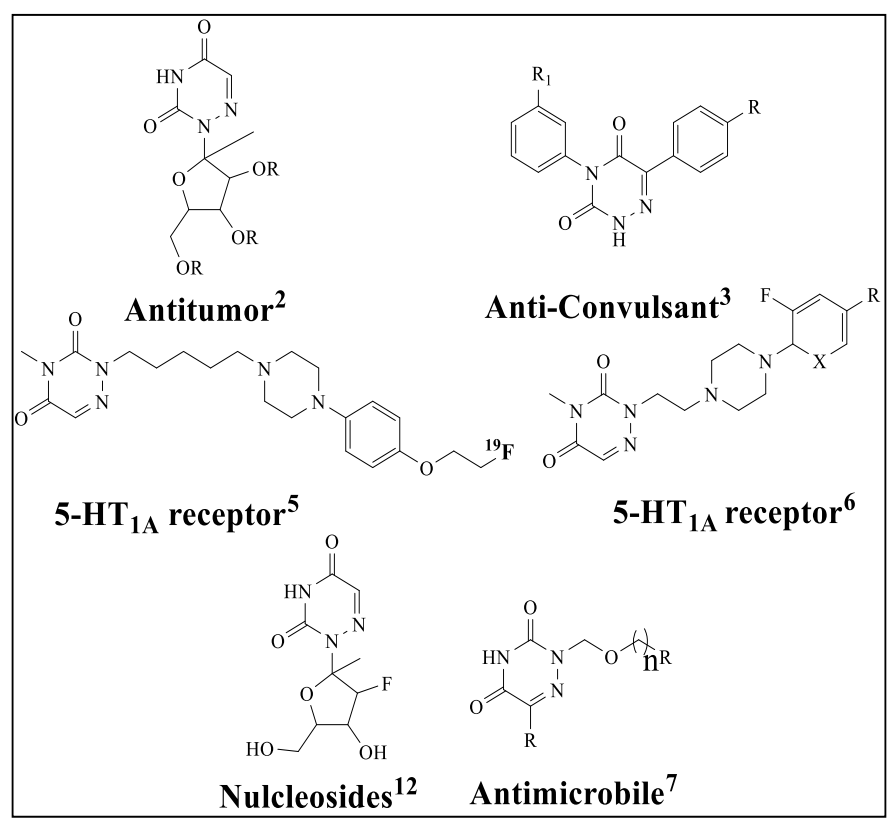

Fig. 1. Some important biological active 6-azauracils. 
Also, ${ }^{1} \mathrm{HNMR}$ spectrum showed the disappearance of both $\mathrm{NH}$ and $\mathrm{OH}$ protons, which confirm that chlorination. A simple nucleophilic attack of removal of chlorine atoms by carbonitrile (DMF), diamino sulfone (DMF), hydrazine hydrate (Fusion) and/or benzene sulfonic acid hydrazide
(DMF), led to the direct formation of 3,5-dicarbonyl-1,2,4triazine (5); 3,5-di(aminosulfonyl amine)-1,2,4-triazine (6); 3,5-dihydrazino-1,2,4-triazine (7) and 3,5-di(benzene sulfonyl hydrazino)-1,2,4-triazine (8) respectively (Scheme 2).

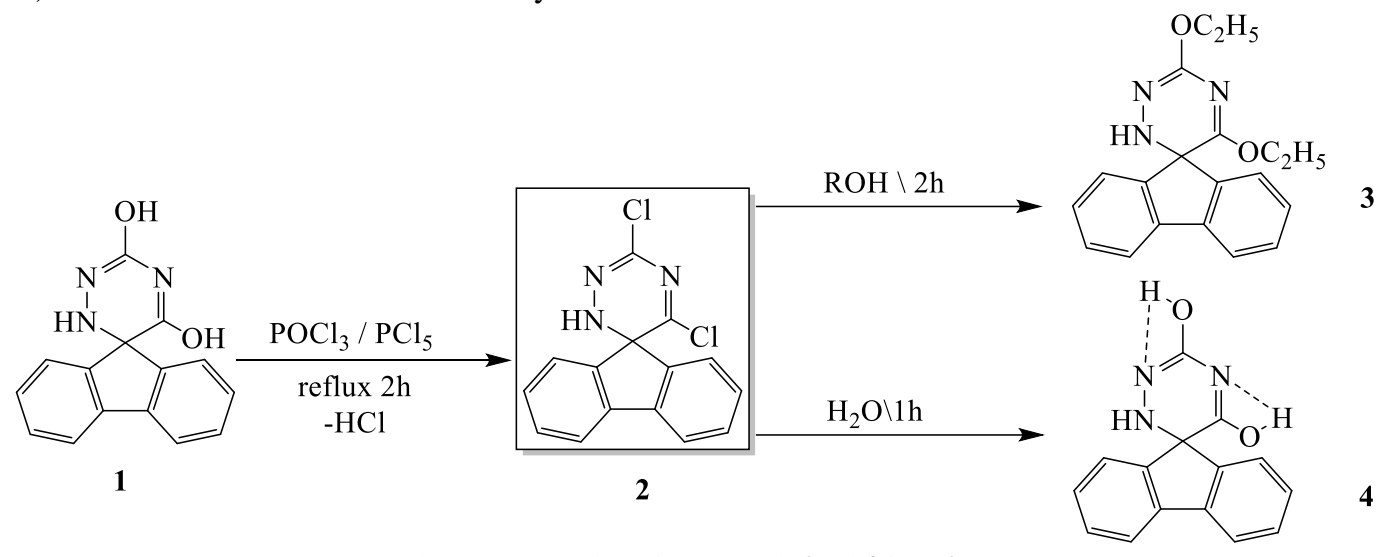

Scheme 1. Formation of compounds $\mathbf{3}$ and $\mathbf{4}$ from $\mathbf{2}$.<smiles>NS(=O)(=O)NC1=NC(=O)C2(NN=C(NS(N)(=O)=O)c3ccccc32)N1</smiles>
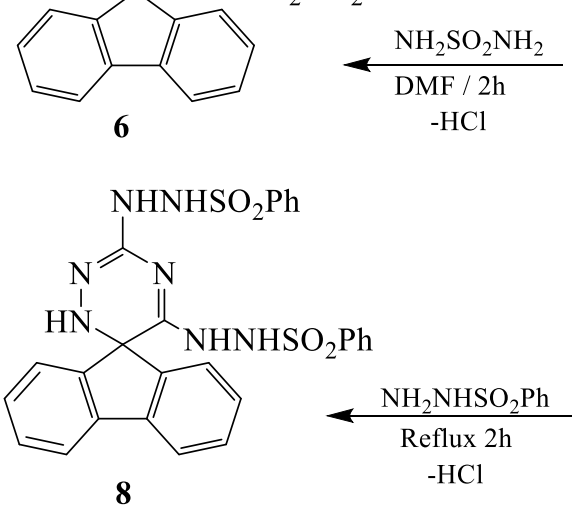

8

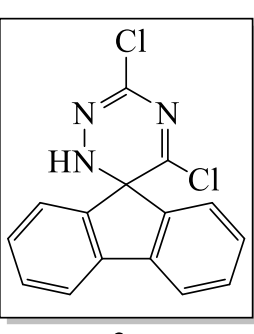

2

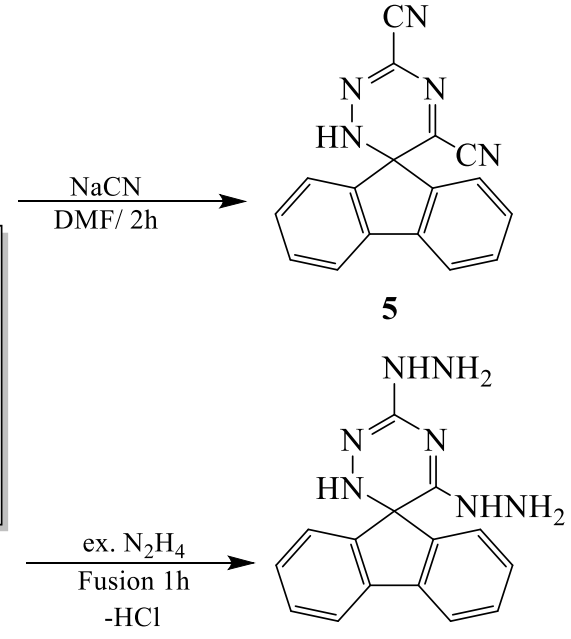

7

Scheme 2. Synthesis of compounds 5-8 from 2.

Structures of compounds $\mathbf{5 - 8}$ can be deduced from their IR spectra, the presence of $v$ at 3100, $2250 \mathrm{~cm}^{-1}(\mathrm{NH}, \mathrm{CN}, \mathbf{5})$, $3350,3150,1350 \mathrm{~cm}^{-1}\left(\mathrm{NH}, \mathrm{NH}_{2}, \mathrm{SO}_{2}, 6\right), 3300,3120 \mathrm{~cm}^{-1}$ $\left(\mathrm{NH}_{2}, \mathrm{NH}, 7\right)$ and $3200,3180,3080,1320 \mathrm{~cm}^{-1}$ (NHNH, NH, $\left.\mathrm{SO}_{2}, 8\right)$. Also, ${ }^{1} \mathrm{HNMR}$ spectra of 5-8 showed a resonated signal at 12.0 and $10.5 \mathrm{ppm}$ for $\mathrm{NH}$ - of 1,2,4-triazine and a side chain. And compounds 6-8 exhibited $\delta$ at $10.8,8.8 \mathrm{ppm}$ for the two $\mathrm{NH}$ of side chain, in addition to $\delta 12 \mathrm{ppm}$ for $\mathrm{NH}$ of 1,2,4-triazine moiety. M/S spectra of 7 recorded the molecular ion peak and the base peak at $165 \mathrm{~m} / \mathrm{z}$ attribute to fluorenyl radical (Fig. 2).

Pyrazoline and pyrazolone derivatives is considered one from a vital unites of biological probes especially if bearing and/or containing 1,2,4-triazine moiety20-24. Thus, the interaction between compound 7 with activated $\pi(-\mathrm{e})$ acceptor carbonitriles as malononitrile (ETOH/piperidine), cyanoacetic acid (ETOH / piperidine) and/or activated methylene compounds as malonic acid $(\mathrm{AcOH})$, afforded the 3,5-di(3',5'-diaminopyrazol-1'-yl)-1,2,4-triazine (9); 3,5di(3'-amino-5'-oxo-4',5'-dihydropyrazol-1'-yl)-1,2,4-triazine
(10), and/or 3,5-di(3',5'-dioxo-4',5'-dihydropyrazol-1'-yl)1,2,4-triazine (11) respectively (Schemes 3 and 4). Formation of compounds 9 and 10 maybe takes place via cycloaddition reaction with $\mathrm{NH} 2$ and $\mathrm{NH}$ of 1,2,4-triazine.

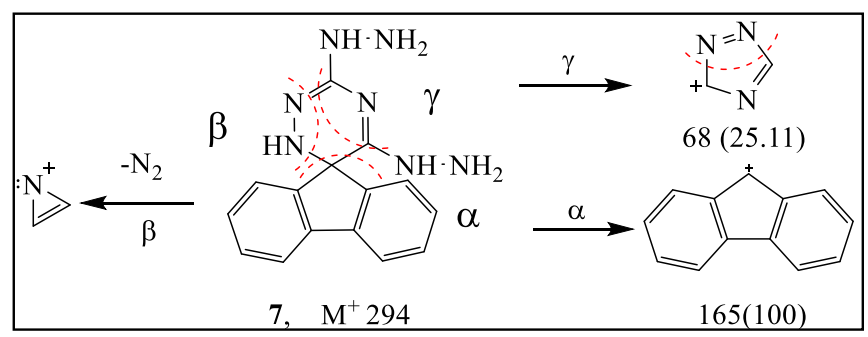

Fig. 2. Mass fragmentation pattern of compound 7.

The structural formula of compounds 9-11 can be established from correct elemental analysis and spectral measurements. IR spectra of compounds 10 and 11 showed both $\boldsymbol{v}$ at 3150, 3100, 1680 1660 and 2980, 2880, 1488, 1440 cm-1 attribute to $\mathrm{NH}, \mathrm{C}=\mathrm{O}$ and $\mathrm{CH} 2$ functional groups, while that of compound 9 recorded only $\boldsymbol{v}$ at 3380 and $3180 \mathrm{~cm}-1$ 
for $\mathrm{NH} 2$ and $\mathrm{NH}$ functional groups. In addition, 1HNMR spectra give us a good indication about their structures. Both, compounds 10 and 11 showed a resonated signal at 13.0, 10.5 and 5 4.66 ppm attributed to $\mathrm{NH}-1,2,4$-triazine, $\mathrm{NH}$ and $\mathrm{CH} 2$ of pyrazole. 1HNMR spectra of 9 recorded $\delta$ at 3.5, 7.8 and $12.8 \mathrm{ppm}$ for $\mathrm{NH} 2, \mathrm{CH}=$ pyrazole and $\mathrm{NH}-1,2,4$-triazine.

Cyclocondensation of 3,5-dihydrazino-1,2,4-triazine 7 with 1,3-bicarbanyl reagents such as, ethyl acetoacetate (THF) and/or diethyl malonate (Dioxane) produced 3,5-di(3'methyl-5'-oxo-4',5'-dihydropyrazol-1'-yl)-1,2,4-triazine $\mathbf{1 2}$ and/or 3,5-di(3',5'-dioxo-tetrahydro-pyrazol-1'-yl)-1,2,4triazine 13 respectively (Scheme 5).Compound 12 only exhibited $\boldsymbol{v}$ at $1700,3150,2980 \mathrm{~cm}^{-1}$ for $\mathrm{C}=\mathrm{O}, \mathrm{NH}$ and $\mathrm{CH}_{2}$, $\mathrm{CH}_{3}$ functional. IR spectrum of recorded only $\mathrm{NH}-$ of 1,2,4triazine with $\mathrm{NH}$ pyrazole and $\mathrm{C}=\mathrm{O}$ functional groups. IR spectrum of 13 recorded only $\mathrm{NH}$ of 1,2,4-triazine with $\mathrm{NH}$ of pyrazole and $\mathrm{C}=\mathrm{O}$ functional groups. ${ }^{1} \mathrm{HNMR}$ spectrum of 12 and 13 showed $\delta$ at $11.88,7.8-6.6$, and $4-2 \& 1.25$ ppm for $\mathrm{NH}, 1,2,4$-triazine aromatic and aliphatic protons. ${ }^{13} \mathrm{C} \mathrm{NMR}$ spectrum of 12 recorded $\delta$ at 160,140, 129-122, 40 and 25 ppm attribute to $\mathrm{C}=\mathrm{O}, \mathrm{C}=\mathrm{N}$, aromatic and aliphatic carbons.

It is interested that reactions of compounds 7 and a cyclic oxygen reagents such as chalcone (ETOH/piperidine) and/or phthalic anhydride $(\mathrm{AcOH})$, gave 3,5-di(3',5'-diphenyl-4',5'dihydropyrazol-1'-yl)-1,2,4-triazine 14 and/or 3,5-di(1',4'phthalazindione-1'-yl)-1,2,4-triazine $\mathbf{1 5}$ respectively (Scheme 6). The structures of the compounds $\mathbf{1 4}$ and $\mathbf{1 5}$ have been deduced from their correct elemental analysis spectral data. IR spectrum of $\mathbf{1 4}$ recorded only $\mathrm{NH}$ of 1,2,4-triazine and $\mathrm{CH}_{2}$ of $\boldsymbol{v}$ at $3100-3080$ and $2970,2890 \mathrm{~cm}^{-1}$ with lacks of $\mathrm{C}=\mathrm{O}$ group, while ${ }^{1} \mathrm{HNMR}$ spectrum showed $\delta$ at $8-6.6$

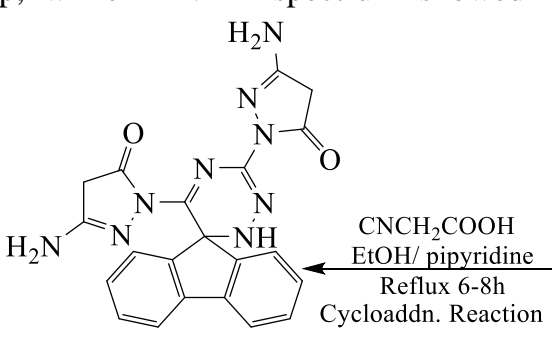

10

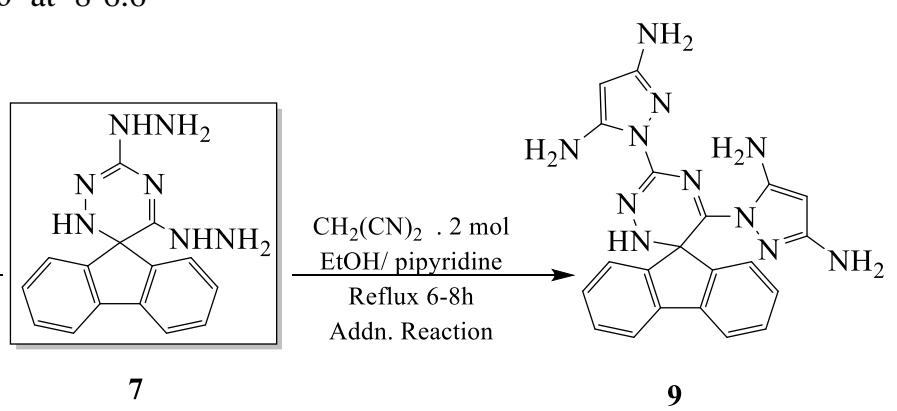

7 (aromatic protons), 5 and $3.8 \mathrm{ppm}$ for $\mathrm{CH}-\mathrm{Ph}$ and $\mathrm{CH}_{2}$ of pyrazole, in addition, $12.8 \mathrm{ppm}$ for NH-1,2,4-triazine. On other hand, IR spectrum of $\mathbf{1 5}$ showed $\boldsymbol{v}$ at 3150, 1700, 1680 and $1600 \mathrm{~cm}^{-1}$ attribute to $\mathrm{NH}, \mathrm{C}=\mathrm{O}, \mathrm{C}=\mathrm{N}$ and $\mathrm{C}=\mathrm{C}$ functional groups. Only ${ }^{13} \mathrm{C}$ NMR spectrum showed $\delta$ at $168,155,142$ and $130-120 \mathrm{ppm}$ for $\mathrm{C}=\mathrm{O}, \mathrm{O}=\mathrm{C}-\mathrm{NH}$ (phthalazine), $\mathrm{C}=\mathrm{N}$ and aromatic carbons.

Finally, treatment of 3,5-dihydrazino-1,2,4-triazine 7 with monochloroacetic acid (DMF) and/or 1,1-dichloroacetic acid (DMF), led to the direct formation of fused triheterocyclic systems 16 and 17 respectively (Scheme 7).

[1-spiro(5'-fluoren-9'-yl)-1,2,4-triazino[4,3-d][1,2,4] triazino[3,2-c][1,2,4]triazine-6,11(13H)dione] (16). Former structure of compounds 16 and 17 established based on correct elemental analysis and their spectral measurements. The difference in structures between compounds $\mathbf{1 6}$ than $\mathbf{1 7}$ can be deduced from both IR and ${ }^{1} \mathrm{HNMR}$ spectra. Only, compound $\mathbf{1 7}$ showed both the $\boldsymbol{v}$ at 3200-3100, 2980, 2890 and $1700,1670 \mathrm{~cm}^{-1}$ for $\mathrm{NH}, \mathrm{CH}_{2}, \mathrm{C}=\mathrm{O}$ functional groups, while that of 16 recorded only $\boldsymbol{v}$ at 3180 and $1710 \mathrm{~cm}^{-1}$ for $\mathrm{NH}$ and $\mathrm{C}=\mathrm{O}$ group. ${ }^{1} \mathrm{HNMR}$ of $\mathbf{1 7}$ showed $\delta$ at 12-8, 11.2,4.5 ppm for $\mathrm{NH}\left(1,2,4\right.$-triazino), $\mathrm{NH}\left(1,2,4\right.$-triaznone), and $\mathrm{CH}_{2}$ protons, in addition $\delta$ at 7.8-6.88 ppm for aromatic protons.

Also, ${ }^{13} \mathrm{C}$ NMR spectrum of 17 recorded $\delta$ at 166, 150, 140 and $40 \mathrm{ppm}$ attribute $\mathrm{CONH}, \mathrm{COCH}_{2}, \mathrm{C}=\mathrm{N}$, aromatic and aliphatic carbons respectively. M/S spectral study of $\mathbf{1 7}$ showed the molecular ion peak and the base peak at m/z 165 attribute to fluorenyl ion (Fig. 4).

Formation of $\mathbf{1 7}$ may be as showed in Figure 5.

Scheme 1. Synthesis of compounds $\mathbf{9}$ and $\mathbf{1 0}$ from $\mathbf{7}$.<smiles>NN=CNC12NN=C(NN)c3ccccc3C1C=CN=C2N</smiles>

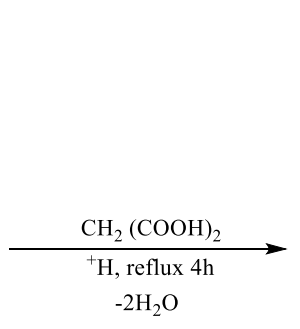<smiles>N=C(NC1NNC(=O)CC1=O)C1(c2ccccc2-c2ccccc2)NC(=O)CC(=O)N1</smiles>

Scheme 2. Formation of compound $\mathbf{1 1}$ from $\mathbf{7}$<smiles>O=C1CC(=O)N(C2=NC(N3NC(=O)CC3=O)C3(N2)c2ccccc2-c2ccccc23)N1</smiles>

13

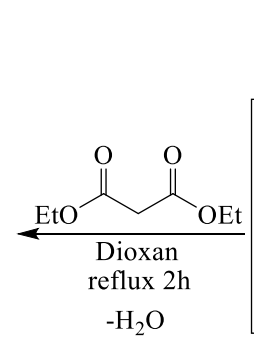

7

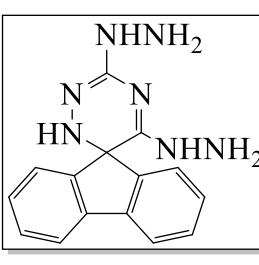

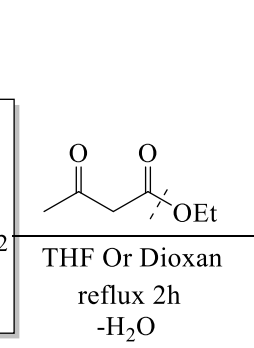

-EtOH

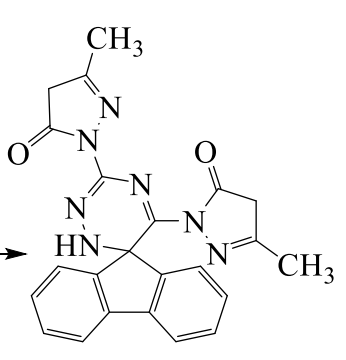

12

Scheme 3. Synthesis of compounds $\mathbf{1 2}$ and $\mathbf{1 3}$ from $\mathbf{7}$. 


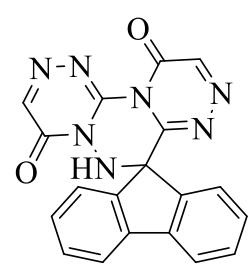

16

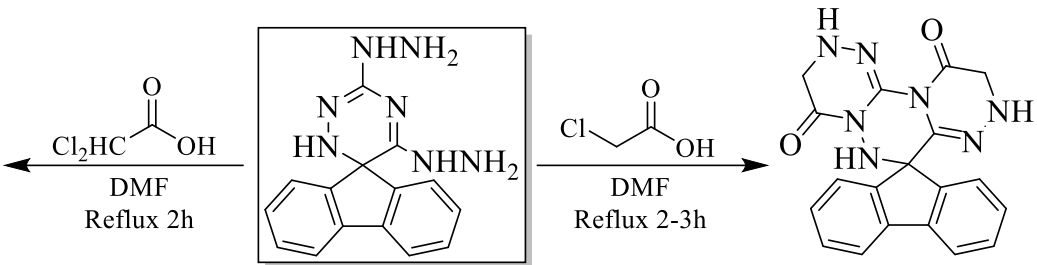

17

Scheme 4. Synthesis of compounds $\mathbf{1 7}$ and $\mathbf{1 8}$ from $\mathbf{7}$

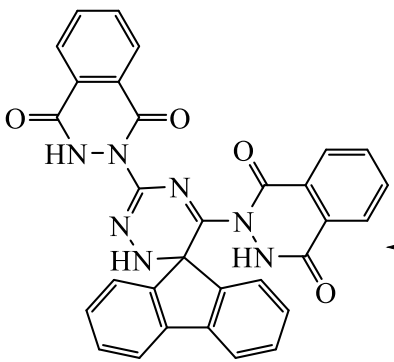

15

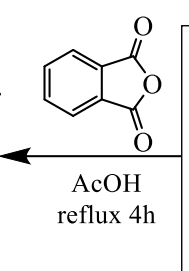

$4 \mathrm{~h}$

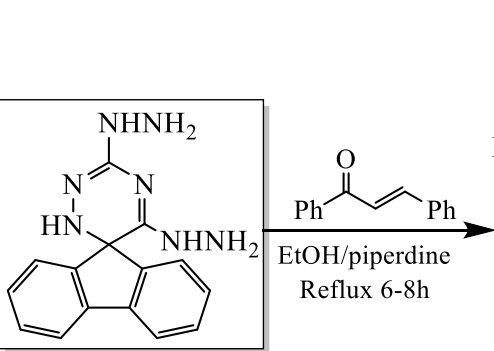

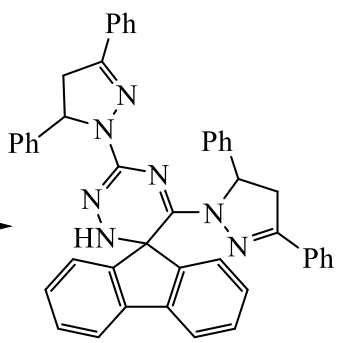

14

Scheme 5. Synthesis of compounds $\mathbf{1 4}$ and $\mathbf{1 5}$ from $\mathbf{7}$.

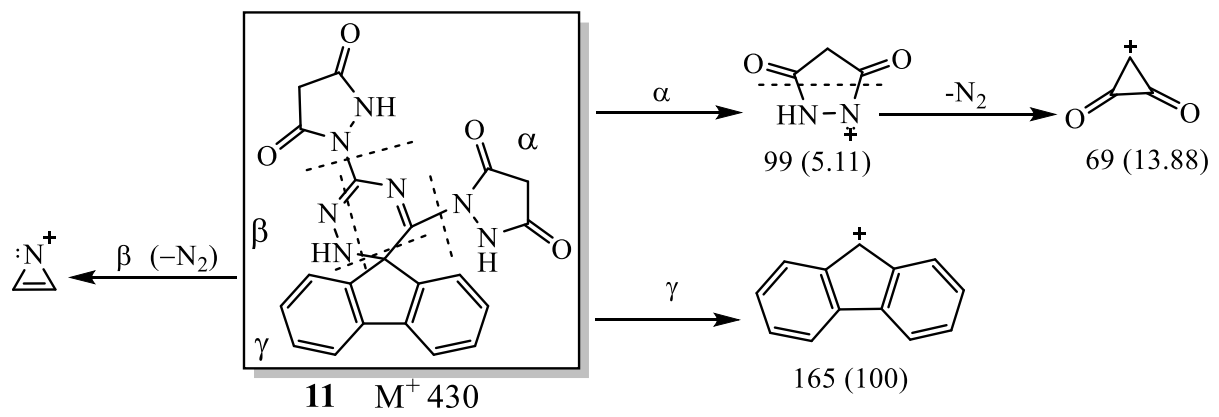

Fig. 3. Mass Fragmentation Pattern of Compound 11.

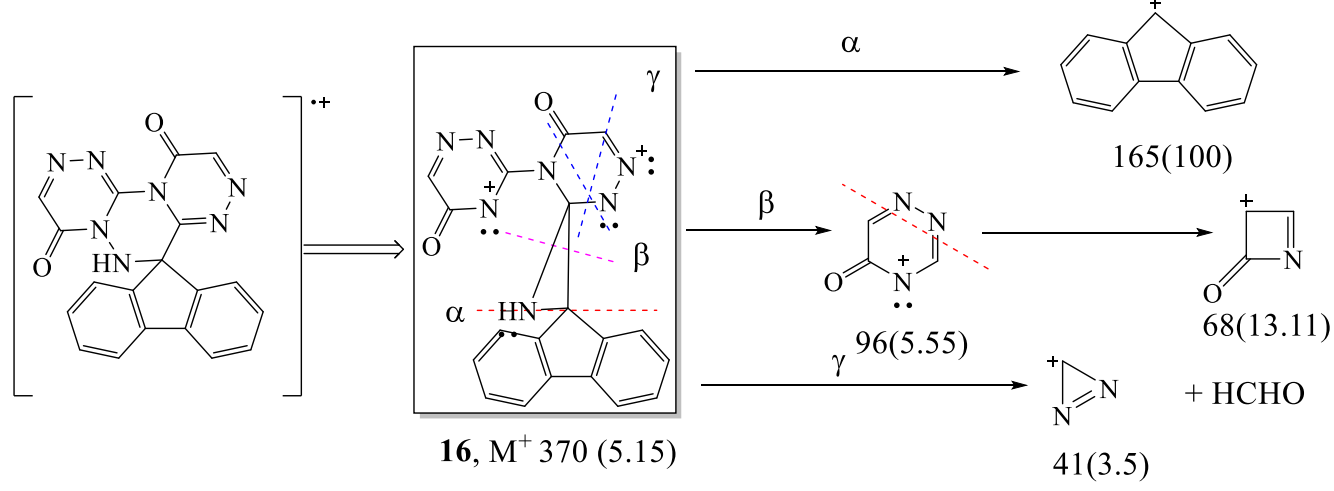

Fig. 4. Mass Fragmentation Pattern of Compound 16.
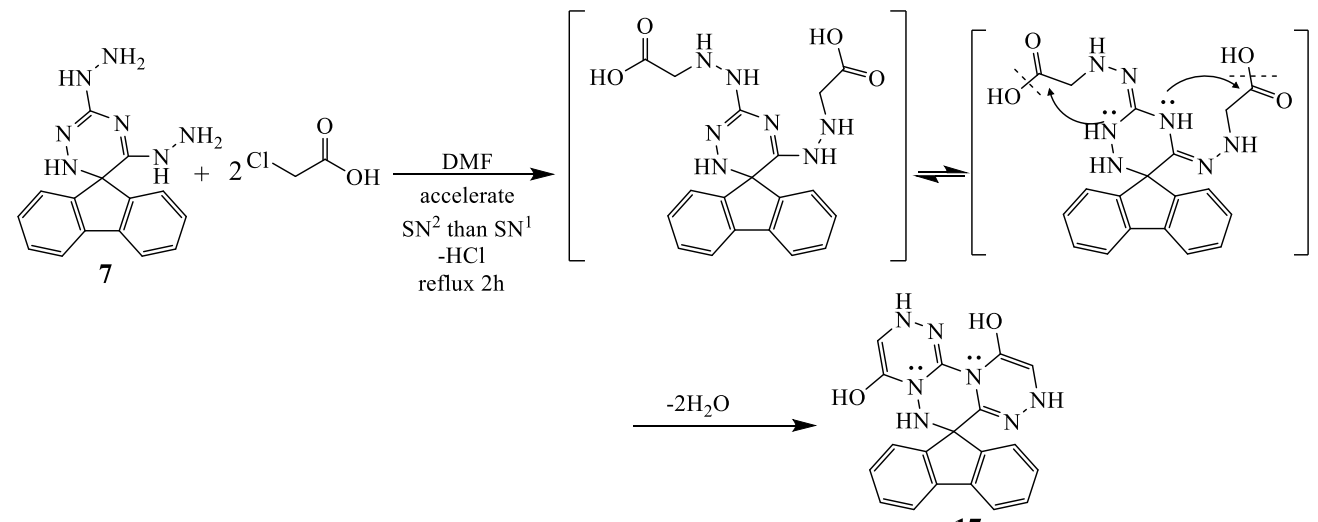

Fig. 5. Mechanism of Compound 7 formation 


\section{THE EXPERIMENTAL}

The commercial chemicals and solvents used in the synthesis were purchased from Sigma-Aldrich Chemical Co. (St. Louis, MO), Fisher Scientific Inc. (Springfield, NJ), or Lancaster (Windham, NH) and were used without further purification. Analytical grade reagents were purchased from standard commercial sources. Melting points determined with an electrothermal Bibly Sturat Scientific melting point sample (UK). A Perkin Elmer Model RXI-FT IR system 55529 was used for recording IR spectra of the prepared compounds. A Bruker advance DPX $400 \mathrm{MHz}$ model uses TMS as internal standard was used for recording the $1 \mathrm{H}$ and 13C NMR spectra of the compounds on deuterated DMSOD6. A GC-MS-GP 1000 Ex model used for recording the mass spectra of the compounds. Electronic spectra recorded in ethanol on Shimadzu UV and visible 310 IPC Spectrophotometer. Elemental analysis was performed in micro analytical center of Cairo University, Cairo, Egypt.

\section{A. 3,5-Dichloro-1H-6-(spiro(fluoren-9'-yl))-6,1-dihydro-} 1,2,4-triazine (2)

A mixture of 1 (0.01 mol), POCl3 (10 ml), and PCl5(5 gm) warmed at $150{ }^{\circ} \mathrm{C}$ for $2 \mathrm{~h}$, cooled then poured part, part on the crutch ice with stirring. The solid obtained filtered off and washed with aq. $\mathrm{Na} 2 \mathrm{CO} 3$, then crystalized from benzene, to give 2 as orange solid, yield $80 \%$, m.p. $240-241 \mathrm{C}$. UV $(\boldsymbol{\lambda} \max$, EtOH): $380 \mathrm{~nm}$. IR(vcm-1): $3120(\mathrm{NH}), 1580(\mathrm{C}=\mathrm{N}), 910$, 850 (aromatic ring), 700(C-Cl). 1HNMR(DMSO-d6) $\delta$ ppm: 12.0( $2,1 \mathrm{H}, \mathrm{NH}$. 1,2,4-triazine), 7.22-7.05 (d, 2H, aromatic), 7.0-6.8 (d, 2H, aromatic), 6.6-6.45 (d, 4H, aromatic).

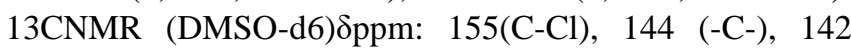
$(\mathrm{C}=\mathrm{N}), 141(\mathrm{C}=\mathrm{N}), 132,130,129,124,123,121$ (aromatic carbons). Anal.Caled; C,59.63; H,3.00; Cl, 23.46, N, 13.91\% for the C15H9Cl2N3(302). Found: C, 59.50; H, 2.88; Cl, $23.19 ; \mathrm{N}, 13.71 \%$.

\section{B. 3,5-Di(ethyloxo)-6-(spiro (fluoren-9'-yl))-6,1-dihydro- 1,2,4-triazine (3)}

A mixture of compound 2 (2gm) and $\mathrm{EtOH}(20 \mathrm{ml})$ refluxed $2 \mathrm{~h}$, cooled, the solid obtained filtered off and crystallized from THF to give 3, as brown solid yield 66\%; m.p. 200-202 C. IR(vem-1): $3150(\mathrm{NH}), 2970,2880($ alkyl $\mathrm{CH})$, 1600 $(\mathrm{C}=\mathrm{C}), 1560(\mathrm{C}=\mathrm{N}), 1480,1420$ (deformation $\mathrm{R}), 1060$ (C-O-C), 880, 810 (aromatic ring). 1HNMR(DMSO-d6)

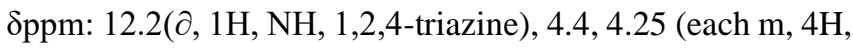
$2 \mathrm{CH} 2), 1.25 \& 1,15$ (each $2,6 \mathrm{H}, 2 \mathrm{CH} 3), 7.6-7.2,7.0-6.8$ \&6.7-6.55 (each d, 8H, aromatic). 13CNMR (DMSO-

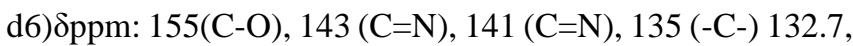
$132.2,130.7,129.8,129.1,128.3,125.1$ (aromatic carbons), 45(CH2), 27(CH3). Anal.Caled; C,70.13; H,7.18; N, 12.91\% for the $\mathrm{C} 19 \mathrm{H} 21 \mathrm{~N} 3 \mathrm{O} 2(326)$. Found: C,70.11; H,7.11; N, $12.80 \%$

\section{C. $\quad$ The tautomeric structure $4 \rightleftharpoons 1$}

Compound 3 (2 gm) and $\mathrm{H}_{2} \mathrm{O}(20 \mathrm{ml})$ refluxed for $1 \mathrm{~h}$, cooled. The solid obtained filtered off and crystalized from benzene to give $\mathbf{4}$ as tautomeric of compound $\mathbf{1}$. The same melting point. But IR spectrum showed a lacks of $\mathrm{OH}$ functional group which confirm that $\mathrm{H}$-bonding formed. UV ( $\lambda$ max EtOH): $280 \mathrm{~nm}$ (hetero conjugated systems).

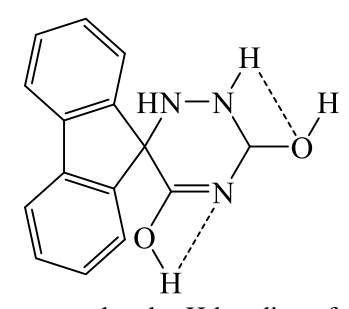

Fig. 6. The exo- and endo- H-bonding of compound 4

D. 3,5-Dicarbnitrile-1,6-dihydro-6-(spiro (fluoren-9'-yl))1,2,4-triazine (5)

A mixture of compound $2(0.01 \mathrm{~mol})$ and $\mathrm{NaCN}(0.02$ $\mathrm{mol}$ ), in few drops $\mathrm{H}_{2} \mathrm{O}$ ) and DMF (20 ml) refluxed $2 \mathrm{~h}$, cooled, then poured onto ice. The solid produced filtered off and crystallized from dioxane to give $\mathbf{5}$ as deep brown solid, yield 70\%; m.p. $210-212^{\circ} \mathrm{C}$. UV ( $\left.\boldsymbol{\lambda}_{\max }, \mathrm{EtOH}\right): 320,270,220$ $\mathrm{nm}(\mathrm{C} \equiv \mathrm{N}, \mathrm{C}=\mathrm{N}, \mathrm{C}=\mathrm{C}) . \operatorname{IR}\left(v \mathrm{~cm}^{-1}\right): 3110(\mathrm{NH}), 2250(\mathrm{C} \equiv \mathrm{N})$, $16810(\mathrm{C}=\mathrm{C}), \quad 1580(\mathrm{C}=\mathrm{N}), 860,810$ (aromatic rings). ${ }^{1} \mathrm{HNMR}\left(\mathrm{DMSO}-\mathrm{d}_{6}\right.$ ) $\delta$ ppm: 11.88, 10.55 (each s, 2H, NH, $\mathrm{NH}$ ), 7.78-7.69, 7.6-7.4, 7.1-6.66 (each $\mathrm{m}, 8 \mathrm{H}$, aromatic). ${ }^{13} \mathrm{CNMR} \quad\left(\mathrm{DMSO}-\mathrm{d}_{6}\right) \quad \delta p p m: 144(\mathrm{C}=\mathrm{N}), 142.2 \quad(\mathrm{C}=\mathrm{N})$, $141.1(\mathrm{C}=\mathrm{N}), 138(-\mathrm{C}-), 133.1,132.5,132.2,131.5,129.9$, 129.8, 129.2, 128.7, 127.9, 127.4, 124.6 (aromatic carbons). Anal.Caled; C,72.08; H,3.20; N, 24.72 for the $\mathrm{C}_{17} \mathrm{H}_{9} \mathrm{~N}_{5}(284)$. Found: C,71.98; H,3.01; N, 24.59\%.

\section{E. 3,5-Di(amino sulfonyl amine)-1,6-dihydro-6-(spiro (fluoren-9'-yl))-1,2,4-triazine (6)}

A mixture of compound $2(0.01 \mathrm{~mol})$ and diamino sulfone $(0.02 \mathrm{~mol})$, in DMF $(20 \mathrm{ml})$ refluxed $2 \mathrm{~h}$, cooled, then poured onto ice. The yielded solid filtered off and crystallized from EtOH to give 6 as dark brown solid, yield 71\%; m.p. 260-262 ${ }^{\circ} \mathrm{C} . \quad \operatorname{IR}\left(v \mathrm{~cm}^{-1}\right): 3350 \quad\left(\mathrm{NH}_{2}\right), \quad 3150(\mathrm{NH}), 1600 \quad(\mathrm{C}=\mathrm{C})$, $1580(\mathrm{C}=\mathrm{N}), 1360\left(\mathrm{SO}_{2} \mathrm{NH}\right), 880,810$ (aromatic rings). ${ }^{1} \mathrm{HNMR}\left(\mathrm{DMSO}-\mathrm{d}_{6}\right.$ ) $\delta$ ppm: 12.1, 10.55 (each s, 2H, 2NH), 3.6, 3.8 (each s, 4H, 2NH2SO2), 7.78-7.69, 7.6-7.4(d, 2H, aromatic), 7.1-6.66 (d, 2H, aromatic), 6.5-6.2(m, 4H, aromatic). ${ }^{13} \mathrm{CNMR}$ (DMSO-d $\left.{ }_{6}\right) \delta \mathrm{ppm}$ : 144.34(NCN), 142.2 $(\mathrm{C}=\mathrm{N}), 141.1(\mathrm{C}=\mathrm{N}), 135(-\mathrm{C}-), 133.6,132.6,132.2,130.7$, 129.9, 129.5, 128.4, 124.6 (aromatic carbons). Anal.Caled; $\mathrm{C}, 42.75 ; \mathrm{H}, 3.59 ; \mathrm{N}, 23.26 ; \mathrm{S}, 15.21$ for the $\mathrm{C}_{15} \mathrm{H}_{15} \mathrm{~N}_{7} \mathrm{O}_{4} \mathrm{~S}_{2}$ (422). Found: C,42.61; H,3.40; N, 23.16; S, 15.00\%.

\section{F. 3,5-Dihydrazino-1,6-dihydro-6-(spiro(fluoren-9'-yl))- 1,2,4-triazine (7)}

A mixture of compound $2(0.01 \mathrm{~mol})$ and hydrazine hydrate $(0.025 \mathrm{~mol})$ refluxed for $1 \mathrm{~h}$ at $200{ }^{\circ} \mathrm{C}$, cooled, then treated with cold $\mathrm{MeOH}$. The solid obtained filtered off and crystallized from EtOH to give 7, as deep red solid, yield $65 \%$; m.p. $<300{ }^{\circ} \mathrm{C} . \operatorname{IR}\left(v \mathrm{~cm}^{-1}\right): 3300\left(\mathrm{NH}_{2}\right), 3120(\mathrm{NH})$, 3050(aromatic $\mathrm{CH}), 1600(\mathrm{C}=\mathrm{C}), 1570(\mathrm{C}=\mathrm{N}), 860.5,840$ (aromatic rings). ${ }^{1} \mathrm{HNMR}\left(\mathrm{DMSO}-\mathrm{d}_{6}\right) \delta \mathrm{ppm}: 11.88(\mathrm{~s}, 1 \mathrm{H}$, $\mathrm{NH}), 10.88$ ( s, 1H, NH), 3.65(s, 2H, $\left.\mathrm{NH}_{2}\right), 7.72-7.66,7.6-$ $7.4(\mathrm{~d}, 2 \mathrm{H}$, aromatic), 7.55-7.45 (d, 2H, aromatic), 7.35-

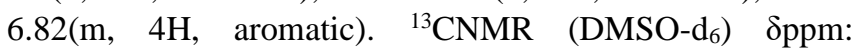
142.34(C=N), $141.1(\mathrm{C}=\mathrm{N}), 135.8(\mathrm{C}=\mathrm{N}), 135.8(-\mathrm{C}-), 132.7$, $132.2,130.8,130.5,129.9,129.51,123.4,123.6$ (aromatic carbons). Anal.Caled; C,61.42; H,5.15; N, 33.43 for the $\mathrm{C}_{15} \mathrm{H}_{15} \mathrm{~N}_{7}$ (294). Found: C, 61.29; H, 5.05; N, 33.20\%. 


\section{G. 3,5-Dibenzenesulfonylhydrazino-1,6-dihydro-6-} spiro(fluoren-9'-yl)-1,2,4-triazine (8)

A mixture of compound 2 ( $0.01 \mathrm{~mol})$ and benzene sulfonic acid hydrazide $(0.02 \mathrm{~mol})$ in DMF $20 \mathrm{ml}$ refluxed for $2 \mathrm{~h}$ at $200{ }^{\circ} \mathrm{C}$, cooled, then poured onto ice. The yielded solid filtered off and crystallized from EtOH to give 8, as yellow powder, yield $60 \%$; m.p. $<300{ }^{\circ} \mathrm{C}$. $\operatorname{IR}\left(v \mathrm{~cm}^{-1}\right): 3200\left(\mathrm{NH}_{2}\right)$, 3180(NH), $3088(\mathrm{NH}), 1580(\mathrm{C}=\mathrm{N}), 1320\left(\mathrm{SO}_{2} \mathrm{NH}\right), 870.5$, 859.6 (aromatic rings). ${ }^{1} \mathrm{HNMR}\left(\mathrm{DMSO}-\mathrm{d}_{6}\right) \delta \mathrm{ppm}: 11.88$ (s, $1 \mathrm{H}, \mathrm{NH}, 1,2,4$-trizine), $8.88-8.55$ (d, 2H,NH-NHSO 2$), 10.88$ ( s, $1 \mathrm{H}, \mathrm{NH}), 3.65\left(\mathrm{~s}, 2 \mathrm{H}, \mathrm{NH}_{2}\right), 7.88-7.77(\mathrm{~m}, 5 \mathrm{H}$, phenyl), 7.55-7.45 (d, 2H, aromatic), 7.4-7.25 (d, 2H, aromatic), 7.16.99 (m, 4H, aromatic). ${ }^{13} \mathrm{CNMR}$ (DMSO-d ${ }_{6}$ ) $\delta$ ppm: 141.19 $(\mathrm{C}=\mathrm{N}), 136.2(\mathrm{C}=\mathrm{N}), 135.8(\mathrm{C}=\mathrm{N}), 135.8(-\mathrm{C}-), 132.7,132.2$, $130.8,130.5,129.9,129.51,123.4,123.6$ (aromatic carbons). Anal.Caled; C,56.53; H,4.05; N, 17.09 S, 11.18 for the $\mathrm{C}_{27} \mathrm{H}_{23} \mathrm{~N}_{7} \mathrm{O}_{4} \mathrm{~S}_{2}$ (574). Found: C,56.42; H,3.89; N, $16.91 \mathrm{~S}$, $11.01 \%$.

\section{H. 3,5-Di(3,5-diaminopyrazol-1'-yl)-1,6-dihydro-6-spiro (fluoren-9'-yl)-1,2,4-triazine (9)}

A mixture of compound $7(0.01 \mathrm{~mol})$ and malononitrile $(0.02 \mathrm{~mol})$ in $\mathrm{EtOH} 50 \mathrm{ml}$, piperidine (drops) refluxed for $8 \mathrm{~h}$, cooled, then poured onto ice. The solid produced filtered off and crystallized from $\mathrm{EtOH}$ to give 9, as deep red powder, yield $80 \%$; m.p. $155-157{ }^{\circ} \mathrm{C}$. IR $\left(v \mathrm{~cm}^{-1}\right): 3380\left(\mathrm{NH}_{2}\right), 3180$ $(\mathrm{NH}), 3010$ (aromatic CH), $1590(\mathrm{C}=\mathrm{N}), 880.5,859.6$ (aromatic rings). ${ }^{1} \mathrm{HNMR}\left(\mathrm{DMSO}-\mathrm{d}_{6}\right) \delta \mathrm{ppm}$ : $11.00(\mathrm{~s}, 1 \mathrm{H}$, $\mathrm{NH}, 1,2,4$-trizine), $7.9(\mathrm{~s}, 1 \mathrm{H}$, cyclic $\mathrm{CH}=\mathrm{N}), 7.78-7.66(\mathrm{~d}$, 2H,aromatic),7.6-7.4 (d, 2H, aromatic), 7.2-6.99 (m, 4H, aromatic), $3.55 \& 3.68$ (each s, $4 \mathrm{H}, 2 \mathrm{NH}_{2}$ ). ${ }^{13} \mathrm{CNMR}$ (DMSO$\left.\mathrm{d}_{6}\right) \delta$ ppm: $160.19\left(\mathrm{C}-\mathrm{NH}_{2}\right), 159.2\left(\mathrm{C}-\mathrm{NH}_{2}\right), 155.18(\mathrm{~N}-\mathrm{C}-\mathrm{N}$ pyrazole $), \quad 142.2(\mathrm{C}=\mathrm{N}), \quad 141.19(\mathrm{C}=\mathrm{N}), \quad 135.2(-\mathrm{C}-)$, 133.99(NN-C-N), 132.68, 132.21, 130.79, 129.5, 129.21, 129.18, 123.4, 123.6 (aromatic carbons). Anal.Caled; C,59.28; H,4.50; N, 36.21 for the $\mathrm{C}_{21} \mathrm{H}_{19} \mathrm{~N}_{11}$ (426). Found: C,59.11; H,4.35; N, 36.01\%.

\section{3,5-Di(3-amino-5-oxo-4,5-dihydropyrazol-1'-yl)-1,6- dihydro-6-spiro (fluoren-9'-yl)-1,2,4-triazine (10)}

A mixture of compound 7 (0.01 mol) and cyanoacetic acid $(0.02 \mathrm{~mol})$ in EtOH $50 \mathrm{ml}$, piperidine (drops) refluxed for $8 \mathrm{~h}$, cooled, then poured onto ice. The solid produced filtered off and crystallized from dioxan to give 10, as redish gum, yield $40 \%$; m.p. $105-107{ }^{\circ} \mathrm{C}$. IR( $\left(v \mathrm{~cm}^{-1}\right): 3150,3100(\mathrm{NH}), 1680 \mathrm{~m}$ 1660 $(\mathrm{C}=\mathrm{O}), \quad 2980, \quad 2880$ (aliphatic $\mathrm{CH}), \quad 1488$, 1440(deformation $\mathrm{CH}_{2}$ ), 860(ArCH). ${ }^{1} \mathrm{HNMR}\left(\mathrm{DMSO}_{6}\right.$ )

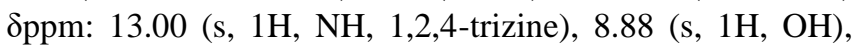
8.20(s, =CH pyrazolo), 7.9-7.72(d, 2H, aromatic), 7.6-7.42 (d, $2 \mathrm{H}$, aromatic), 7.2-6.83 (m, 4H, aromatic). ${ }^{13} \mathrm{CNMR}$ (DMSO$\left.\mathrm{d}_{6}\right) \delta$ ppm: 161.26, 159.50( (C=O), 156.2, 155(C-NH$), 142.2$, 141.1, 139.6, $139.0(\mathrm{C}=\mathrm{N}), 135.82(-\mathrm{C}-), 132.69,131.68$, $130.21,130.79,129.88,129.80,128.81,123.4,123.6$ (aromatic carbons), 120.23, 119.98( C5-C6 1,2,4-triazine), 40.11 $\left(\mathrm{CH}_{2}\right)$. Anal.Caled; C,59.01; H,4.01; N, 29.49 for the $\mathrm{C}_{21} \mathrm{H}_{17} \mathrm{~N}_{9} \mathrm{O}_{2}$ (428). Found: C,58.89; H,3.88; N, $29.31 \%$.

J. 3,5-Di(3,5-dioxo-1,2,3,4-tetrahydropyrazol-1'-yl)-1,6dihydro-6-spiro (fluoren-9'-yl)-1,2,4-triazine (11)

A mixture of compound $7(0.01 \mathrm{~mol})$ and malonic acid $(0.02 \mathrm{~mol})$ in glacial AcOH $50 \mathrm{ml}$, refluxed for $4 \mathrm{~h}$, cooled, then poured onto ice. The solid produced filtered off and crystallized from THF to give 11, as brown gum, yield 70\%; m.p. $\quad 112-114{ }^{\circ} \mathrm{C} . \quad \operatorname{IR}\left(v \mathrm{vcm}^{-1}\right): \quad 3300\left(\mathrm{NH}_{2}\right), \quad 3120(\mathrm{NH})$, $1680(\mathrm{C}=\mathrm{O}), \quad 1580(\mathrm{C}=\mathrm{N}), \quad 860$ (aromatic rings).

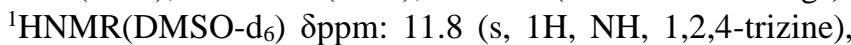
$8.56,8.66$ (each s, $2 \mathrm{H}, 2 \mathrm{NH}), 4.5,4.35$ (each s, $4 \mathrm{H}, 2 \mathrm{CH}_{2}$ ), 7.78-7.74(d, $2 \mathrm{H}$, aromatic), 7.6-7.42 (d, 2H, aromatic), 7.26.83 (m, 4H, aromatic). ${ }^{13} \mathrm{CNMR}$ (DMSO-d 6 ) $\delta$ ppm: 157.72, 155.50 $(\mathrm{C}=\mathrm{O}), 144.61,144.39,143.50,143.4(\mathrm{C}=\mathrm{N}), 142.62$, 142.26, 141.56, 141.19 (C-N), 135.72(-C-), 132.69, 132.21, $130.90,130.79,129.96,129.83,128.80,129.75,129.55$, 129.46, 129.40, 129.18 (aromatic carbons), 40.04( $\left.\mathrm{CH}_{2}\right)$. Anal.Caled; C,58.74; H,3.52; N, 22.83 for the $\mathrm{C}_{21} \mathrm{H}_{15} \mathrm{~N}_{7} \mathrm{O}_{4}$ (430). Found: C,58.61; H,3.38; N, 22.70.

\section{K. 3,5-Di(3-methyl-5-oxo-4,5-dihydropyrazol-1'-yl)-1,6- dihydro-6-spiro (fluoren-9'-yl)-1,2,4-triazine (12)}

A mixture of compound $7(0.01 \mathrm{~mol})$ and ethyl acetoacetate $(0.02 \mathrm{~mol})$ in THF $(100 \mathrm{ml})$, refluxed for $2 \mathrm{~h}$, cooled, then poured onto ice. The solid produced filtered off and crystallized from dioxane to give 12, as brown powder, yield $79 \%$; m.p. $280-282{ }^{\circ} \mathrm{C}$. IR $\left(v \mathrm{~cm}^{-1}\right): 3150,3100(\mathrm{NH})$, 2960, 2888(aliphatic CH), 3020 (aromatic CH), 1700(C=O), 1489, 1420(deformation $\mathrm{CH}_{2}$ ), 860, 822(aromatic rings) ${ }^{1} \mathrm{HNMR}\left(\mathrm{DMSO}-\mathrm{d}_{6}\right)$ Sppm: 11.88 (s, 1H, NHP), 4.25(t,2H, $\left.\mathrm{CH}_{2}\right)$ 4.55(t, 2H, $\left.\mathrm{CH}_{2}\right), 1.23\left(\mathrm{~s}, 3 \mathrm{H}, \mathrm{CH}_{3}\right), 1.03\left(\mathrm{~s}, 3 \mathrm{H}, \mathrm{CH}_{3}\right)$. ${ }^{13} \mathrm{CNMR}\left(\mathrm{DMSO}-\mathrm{d}_{6}\right) \delta \mathrm{ppm}$ : 155.2(C=O), $142.26(\mathrm{C}=\mathrm{N})$, 141.50, 141.19(C=N), 135.85(-C-), 132.69, 132.48, 130.21, 130.79, 129.88, 129.80, 128.81, 123.4, 123.6 (aromatic carbons), 40.05( $\left(\mathrm{CH}_{2}\right)$. Anal.Caled; C,64.94; H,4.47; N, 23.05 for the $\mathrm{C}_{23} \mathrm{H}_{19} \mathrm{~N}_{7} \mathrm{O}_{2}$ (425). Found: $\mathrm{C}, 64.80 ; \mathrm{H}, 4.29 ; \mathrm{N}$, $22.85 \%$.

\section{3,5-Di(3,5-dioxo-tetrahydropyrazol-1'-yl)-1,6-dihydro- 6-spiro(fluoren-9'-yl)-1,2,4-triazine (13)}

A mixture of $7(0.01 \mathrm{~mol})$ and diethylmalonate $(0.02 \mathrm{~mol})$ in dioxin $(50 \mathrm{ml})$ refluxed $2 \mathrm{~h}$ and cooled. The solid obtained, filtered off and crystallized from $\mathrm{EtOH}$ to give 13, as offwhite powder, yieled $74 \%$, m.p. $204-205{ }^{\circ} \mathrm{C}$. IR $\left(\mathrm{vm}^{-1}\right)=3150$ $(\mathrm{NH}), 2980,2860$ (aliphatic CH), 1680,1660, $1590(\mathrm{C}=\mathrm{N})$, $880,860,810$ (aromatic vinyls). ${ }^{1} \mathrm{HNMR}$ (DEMSO-d6) $\delta \mathrm{ppm}=11.8(\mathrm{~s}, 1 \mathrm{H}, \mathrm{NH}) 8.8-8.5(\mathrm{~s} .2 \mathrm{H}, \mathrm{NH}) 7.88-7.78(\mathrm{~d}, 2 \mathrm{H}$, aromatic) 7.66-7.59 (d, 2H, aromatic) 7.4-6.99 (m, 4H, aromatic) 4.55-4.42 (each d, d, 4H, 2 $\mathrm{CH}_{2}$ ). ${ }^{13} \mathrm{CNMR}$ (DMSOd6) $\delta p p m=155.14(\mathrm{C}=\mathrm{O}), 142.26,141.19(\mathrm{C}=\mathrm{N}), 135.86$ (aliphatic $\mathrm{CH}_{4}$ ), 132.69, 136.22, 130.79, 129.97, 129.80, 129.25, 129.18 (aromatic carbons) 40.11, $39.65\left(\mathrm{CH}_{2}\right)$. Anal.Caled; C,58.74; H,3.49; N, 22.04 for the $\mathrm{C}_{21} \mathrm{H}_{15} \mathrm{~N}_{7} \mathrm{O}_{4}$ (429). Found: C,58.80; H,3.39; N, 22.69\%.

\section{3,5-Di(3,5-diphenyl-4,5-dihydropyrozol-i-yl)-1,6- dihydro-6-spiro(fluoren -9-yl)-1,2,4-trinzine (14)}

A mixture of $7(0.01 \mathrm{~mol})$ and chalcone $(0.02 \mathrm{~mol})$ in $\mathrm{EtOH}$ $(50 \mathrm{ml})$, with piperidine (drops) refluxed $8 \mathrm{~h}$ cooled then poured onto ice. The yielded solid filtered off and crystallized from EtOH, to give 14, as red powder, yield 70\%, m.p. 195$197{ }^{\circ} \mathrm{C}$. IR $\left(\mathrm{vm}^{-1}\right)$ : 3100, $3088(\mathrm{NH}), 2970,2890$ (aliphatic $\mathrm{CH}), 1600(\mathrm{C}=\mathrm{C}), 1570(\mathrm{C}=\mathrm{N}), 860-840$ (aromatic rings). ${ }^{1} \mathrm{HNMR} \quad$ (DMSO,d6) $\quad \delta p p m: \quad 12.8(\mathrm{~s}, 1 \mathrm{H}, \mathrm{NH})$, 8.2(s,1H,aromatic), 7.8-7.66 (d, 2H,aromatic) 7.4-7.3 (d, 2H, aromatic), 7.2-6.89(m, $4 \mathrm{H}$, aromatic), 5.5(s,1H,CH-phenyl), 
3.8(s, 2H, $\left.\mathrm{CH}_{2}\right) .{ }^{13} \mathrm{CNMR}$ (DEMSO-d6) $\delta \mathrm{ppm}=142.36$, $141.19(\mathrm{C}=\mathrm{N})$ 135.86(-C-), 132.7, 132.2, 130.7, 129.8, 129.2, 129.1, 123.5(aromatic carbons), 121.5-121.35(C-C of pyrazole), $40.5\left(\mathrm{CH}_{2}\right)$. Anal.Caled; $\mathrm{C}, 80.21 ; \mathrm{H}, 5.24 ; \mathrm{N}, 14.55$ for the $\mathrm{C}_{45} \mathrm{H}_{45} \mathrm{~N}_{7}$ (674). Found: C,79.89; H,5.11; N, $14.35 \%$.

N. 3,5-Di(1,4-dioxo-phthalazine-2'-yl)-1,6-dihydro-6spiro(fluoren-9'-yl)-1,2,4-triazine (15)

A mixture of $7(0.01 \mathrm{~mol})$ and phthalic anhydride $(0.02$ $\mathrm{mol})$ in glacial $\mathrm{A}_{2} \mathrm{OH}(50 \mathrm{ml})$. refluxed $4 \mathrm{~h}$, cooled then poured onto ice. The obtained solid, filtered off and crystallized from EtOH to give 15, as yellow powder, yield 82\%, m.p. $130-132{ }^{\circ} \mathrm{C}$, IR $\left(v^{-1}\right): 3150(\mathrm{NH}), 1700,1680$ $(\mathrm{C}=\mathrm{O}), 1600(\mathrm{C}=\mathrm{C}), 1570(\mathrm{C}=\mathrm{N}), 880,810$ (aromatic rings). ${ }^{1} \mathrm{HNMR}$ (DMSO,d6) $\delta$ ppm: 11.88 (s, 1H, NH, 1,2,4 triazine), $8.88,8.68$ (each d, 2H, 2NH, phthalazine), $7.88-7.76,7.74-$ 7.68 (each $\mathrm{m}, 8 \mathrm{H}$, benzophthalazine), 7.66-7.48 (d, 2H, aromatic), 7.4-7.2 (d, 2H, aromatic) 7.0-6.8 (m,4H, aromatic).

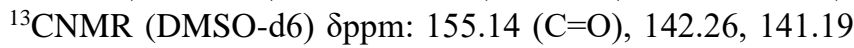
$(\mathrm{C}=\mathrm{N}), 135.86$ (aliphatic $\left.\mathrm{CH}_{4}\right), 132.70,132.22,130.79$, 129.80, 129.2, 129.1 (aromatic carbons), 121.52, 121.35 (carbons of phthalazine). Anal.Caled; C,67.27; H,3.46; $\mathrm{N}, 17.71$ for the $\mathrm{C}_{31} \mathrm{H}_{19} \mathrm{~N}_{7} \mathrm{O}_{4}$ (554). Found: C,67.07; H,3.36; $\mathrm{N}, 17.51 \%$.

\section{O. Fused heterotricyclic 1,2,4-triazinone (16)}

A mixture of $7(0.01 \mathrm{~mol})$ and 1,1'-dichloroacetic acid $(0.02 \mathrm{~mol})$ in DMF $(50 \mathrm{ml})$. refluxed $2 \mathrm{~h}$, cooled then poured onto ice. The solid obtained filtered off and crystallized from EtOH to give 16, as brown powder, yield 30\%, m.p. 175-177 ${ }^{\circ} \mathrm{C}$. UV $\left(\lambda_{\max }\right): 350 \mathrm{~nm}$. IR $\left(v \mathrm{vm}^{-1}\right): 3110(\mathrm{NH}), 3050$ (aromatic $\mathrm{CH}), 1700,1690(\mathrm{C}=\mathrm{O}), 1610(\mathrm{C}=\mathrm{C}), 1580(\mathrm{C}=\mathrm{N}), 890,860$, 810 (aromatic rings). ${ }^{1} \mathrm{HNMR}$ (DMSO,d6) $\delta$ ppm: 11.88 (s, $1 \mathrm{H}, \mathrm{NH}, 1,2,4$ triazine), $8.88,8.68$ (each d, $2 \mathrm{H}, 2 \mathrm{NH}$, $2 \mathrm{CH}=\mathrm{N}, 1,2,4-$ phthalazine), $7.8-7.6(\mathrm{~d}, 2 \mathrm{H}$, aromatic), 7.6$6.68(\mathrm{~m}, 4 \mathrm{H}$, aromatic), 7.4-7.2 (d, 2H, aromatic) 7.0-6.8 (m,4H, aromatic). ${ }^{13} \mathrm{CNMR}$ (DMSO-d6), $\delta$ ppm: 155.14 $(\mathrm{C}=\mathrm{O}), 144.36(\mathrm{C}=\mathrm{N}), 142.26(\mathrm{C}=\mathrm{N}), 141.19(\mathrm{C}=\mathrm{N}), 135.14$ (aliphatic $\left.\mathrm{CH}_{4}\right), 133.69,132.69,132.22,130.79,129.79$, 129.81, 129.25, 129.18 (aromatic carbons), 124.42, 123.21, 121.7, 121.35 (tricyclic carbons). Anal.Caled; C,61.79; $\mathrm{H}, 3.00 ; \mathrm{N}, 26.55$ for the $\mathrm{C}_{19} \mathrm{H}_{11} \mathrm{~N}_{7} \mathrm{O}_{2}$ (370). Found: C,61.67; $\mathrm{H}, 3.36$; N,26.33\%.

\section{P. Fused heterotricyclic perhydro-1,2,4-triazinone (17)}

A mixture of 7 (0.01 mol) and monochloroacetic acid (0.02 $\mathrm{mol})$ in DMF $(20 \mathrm{ml})$. refluxed for $2 \mathrm{~h}$, cooled then poured onto ice. The solid yielded filtered off and crystallized from EtOH to give 17, as deep yellow powder, yield 44\%, m.p. $188-190{ }^{\circ} \mathrm{C}$. IR $\left(v \mathrm{~m}^{-1}\right): 3120(\mathrm{NH}), 3055$ (aromatic $\left.\mathrm{CH}\right)$, 2985, 2890 (aliphatic CH), $1670(\mathrm{C}=\mathrm{O}), 1580(\mathrm{C}=\mathrm{N}), 1580$ $(\mathrm{C}=\mathrm{N}$ ), 1470, 1440 (deformation $\mathrm{CH}$ ), 860, 810 (aromatic rings). ${ }^{1} \mathrm{HNMR}$ (DMSO,d6) $\delta$ ppm: 12.2 and 11.80 (each s, $2 \mathrm{H}, 2 \mathrm{NH}), 7.8-7.6(\mathrm{~d}, 2 \mathrm{H}$, aromatic), 7.5-7.3 (d, 2H, aromatic), 7.1-6.8 (m, 4H, aromatic). ${ }^{13} \mathrm{CNMR}$ (DMSO-d6), Sppm: $150.14(\mathrm{C}=\mathrm{O}), 144.26,142.06,141.8(\mathrm{C}=\mathrm{N}), 135.86(-$ C-), 132.22, 132.69, 132.22, 130.79, 129.79, 129.81, 129.25, 129.18, 124.42, 123.21, 121.78, 121.25 (carbons of tricyclic). Anal.Caled; C,61.12; H,4.05; N,26.26 for the $\mathrm{C}_{19} \mathrm{H}_{15} \mathrm{~N}_{7} \mathrm{O}_{2}$ (374). Found: C,60.90; H,3.95; N,26.13\%.

\section{The ANTIOXIDATION Evaluation}

A substance that are able to neutralize reactive molecules and reduce oxidative damage are called antioxidants. The anti-oxidant substances, prevents the transfer of electron form $\mathrm{O}_{2}$ to the organic molecules, stabilizes free radicals and terminates free radical reactions. Oxidative damage to DNA proteins and other macromolecules has been implicated in the pathogenesis of a wide variety of diseases most notably heart diseases and cancer ${ }^{25}$. In addition, antioxidants intake can help providing added protection for the body against Heart problems, Eye problems, Memory problems, mood disorders and immune system problems ${ }^{26}$. Based upon these observations the antioxidants evaluation of the properad new compounds: 1-1-Diphenyl-2-picryl hydrazyl (DppH) use to produce and reduce the odd electron stable free radical which showed a strong UV absorption maximum at $\lambda \max 517 \mathrm{~nm}$. The New Compounds obtained dissolved in DMSO /EtOH at 150 and $300 \mathrm{mmol}$. $\mathrm{L}^{-1}$ added to DPPH at $100 \mathrm{mmol}$. L ${ }^{-1}$. The tube kept at room temperature for 20 minutes and the absorption measured at $\lambda \max 517 \mathrm{~nm}$. The difference between the test and the control taken as the percent scavenging of the DPPH radical by use the formula:

$$
\% \text { inhibition }=A B-A A /_{A B} \times 100
$$

where $A B=$ Absorption of blank.

$A A=$ absorption of the tested compound.

The radical scavenging activity of ascorbic acid also measured compared with that of the different synthesized compounds ${ }^{27}$. the observed data on the antioxidant activated of the compounds and control shown in Table 1. from the result obtained we can conclude that:

- The activity of the tested compounds increases in the order $11>10>8>12>>17>3$.

- A higher activity of compound 11, 10 and $\mathbf{8}$ may be due to presence of two hydroxy group of pyrazolone and 1,2,4-triazine.

- A lower activity of compounds 17 attribute to a conjugated fused heterocyclic system which had a highly stability with lower electron mobilities. In general, the activity of all the tested compounds based on the activity of 1,2,4 triazine and fluorene moieties.

TABLE I: THE DDH RADICAL SCARVENGING ACTIVITY OF THE NOVEL POLY HETEROCYCLIC NITROGEN SYSTEMS AT 150 AND 300 MMOL. L-1

\begin{tabular}{ccc}
\hline \hline \multirow{2}{*}{ Compound No. } & \multicolumn{2}{c}{ DPPH \%inhibition anti-oxidant $\mp$ SD } \\
$150 \mathrm{mmol}^{-1}$ & $300 \mathrm{mmol}^{-1} \mathrm{~L}^{-1}$ \\
\hline 2 & $50.01 \mp 0.1$ & $50.9 \mp 0.8$ \\
3 & $52.6 \mp 0.2$ & $60.7 \mp 0.3$ \\
6 & $51.6 \mp 0.2$ & $53.3 \mp 0.1$ \\
8 & $60.01 \mp 0.1$ & $63.2 \mp 0.05$ \\
10 & $62.3 \mp 0.1$ & $64.8 \mp 0.1$ \\
11 & $72.0 \mp 0.1$ & $75.5 \mp 0.1$ \\
12 & $53.0 \mp 0.1$ & $57.5 \mp 0.1$ \\
15 & $50.0 \mp 0.7$ & $51.7 \mp 0.8$ \\
16 & $51.8 \mp 0.1$ & $52.3 \mp 0.8$ \\
17 & $56.8 \mp 0.2$ & $61.8 \mp 0.3$ \\
Ascorbic acid & 43.00 & 50.70 \\
\hline \hline
\end{tabular}




\section{CONCLUSION}

In search for some more new antioxidant agent's novel poly heterocyclic nitrogen systems bearing $1,2,4$, triazine moiety derived from 6-azauracy deduced via chlorination and hydrazinolysis followed by ring closure reactions with bfunctional agents in different medium and conditions. The antioxidation accident evaluation indicated that the compounds containing more hydroxyl groups as pyrazole and 1,2,4- triazine exhibited a high to moderate activity towards scavenging of free radical within the body which prevent diseases.

Acknowledgement: Authors would like to thanks Prof. Reda Abdul-Rahman (DSC) for his essential help and constant support.

\section{REFERENCES}

[1] Mary, Y., Al-Tamimi, A., El-Brollosy, N., El-Emam, A., Jojo, P., Panicker, C. and Alsenoy, C. Molecular structure and vibrational spectra of 2-Ethoxymethyl-6-ethyl-2,3,4,5-tetrahydro-1,2,4-triazine3,5-dione, a potential chemotherapeutic agent, by density functional methods. Spectrochimica Acta Part A: Molecular and Biomolecular Spectroscopy, 2014, 133, 439-448.

[2] Harutyunyan, A., Panosyan, H., Tamazyan, R., Aivazyan, A. and Danagulyan, G. 4-alkylated 2-(2,3,5-tri-O-acyl- $\beta$-D-ribofuranosyl)and 2-(2,3,4,6-tetra-O-acetyl- $\beta$-D-glucopyranosyl)-1,2,4-triazine-3,5diones. Russian Journal of Organic Chemistry, 2017, 53(4), 573-576.

[3] Khan, A., Siddiqui, N., Akhtar, M., Ali, Z. and Yar, M. Design, Synthesis, and Biological Evaluation of 6-(2-Amino-substituted phenyl)-4-(substituted phenyl)-1,2,4-triazine-3,5(2H,4H)-dione Derivatives as Anticonvulsant Agents. Archiv der Pharmazie, 2016, 349(4), 277-292.

[4] Hin, N., Duvall, B., Ferraris, D., Alt, J., Thomas, A., Rais, R., Rojas, C., Wu, Y., Wozniak, K., Slusher, B. and Tsukamoto, T. 6-Hydroxy1,2,4-triazine-3,5(2H,4H)-dione Derivatives as Novel d-Amino Acid Oxidase Inhibitors. Journal of Medicinal Chemistry, 2015, 58(18), 7258-7272.

[5] Majo, V., Milak, M., Prabhakaran, J., Mali, P., Savenkova, L., Simpson, N., Mann, J., Parsey, R. and Kumar, J. Synthesis and in vivo evaluation of [18F]2-(4-(4-(2-(2-fluoroethoxy)phenyl)piperazin-1yl)butyl)-4-methyl-1,2,4-triazine-3,5(2H,4H)-dione ([18F]FECUMI$101)$ as an imaging probe for 5-HT1A receptor agonist in nonhuman primates. Bioorganic \& Medicinal Chemistry, 2013, 21(17), 55985604.

[6] Kumar, J., Majo, V., Prabhakaran, J. and Mann, J. Synthesis and evaluation of arylpiperazines derivatives of 3,5-dioxo- $(2 \mathrm{H}, 4 \mathrm{H})-1,2,4$ triazine as 5-HT 1A R ligands. Bioorganic \& Medicinal Chemistry Letters, 2014, 24(19), 4759-4762.

[7] El-Brollosy, N. Synthesis and antimicrobial evaluation of 6-azauracil non-nucleosides. Monatshefte für Chemie - Chemical Monthly, 2008, 139(12), 1483-1490.

[8] Malagon, F., Kireeva, M., Shafer, B., Lubkowska, L., Kashlev, M. and Strathern, J. Mutations in the Saccharomyces cerevisiae RPB1Gene Conferring Hypersensitivity to 6-Azauracil. Genetics, 2006, 172(4), 2201-2209.

[9] Buchtik, R., Slouka, J. and Hlavac, J. Polycyclic heterocycles with acidic N-H group VII. Synthesis of some polynuclear heterocyclic compounds derived from 5-phenyl-6-azauracile. Arkivok, 2006, 2006(5), 78-85.

[10] Bilek, P. and Slauka, J. 5-Substituted 6-Azauracils XVI- Synthesis Of Some Nitrogen Functional Derivatives Of 6-Azauracil-5-Carboxylic Acid. Acta Univ. Palacki. Olomouc., Fac. Rerum. Nat, 2000, 39(37), 7-13.

[11] Seela, F. and Chittepu, P. 6-Azauracil or 8-aza-7-deazaadenine nucleosides and oligonucleotides: the effect of 2 '-fluoro substituents and nucleobase nitrogens on conformation and base pairing. Organic \& Biomolecular Chemistry, 2008, 6(3), 596-607.

[12] Watanabe, K., Reichman, U., Hirota, K., Lopez, C. and Fox, J. Nucleosides. 110. Synthesis and antiherpes virus activity of some 2'fluoro-2'-deoxyarabinofuranosylpyrimidine nucleosides. Journal of Medicinal Chemistry, 1979, 22(1), 21-24.
[13] Watanabe, K., Su, T., Klein, R., Chu, C., Matsuda, A., Chun, M., Lopez, C. and Fox, J. Nucleosides. 123. Synthesis of antiviral nucleosides: 5-substituted 1-(2-deoxy-2-halogeno-.beta.-Darabinofuranosyl)cytosines and -uracils. Some structure-activity relationships. Journal of Medicinal Chemistry, 1983, 26(2), 152-156.

[14] Watanabe, K., Su, T., Reichman, U., Greenberg, N., Lopez, C. and Fox, J. Nucleosides. 129. Synthesis of antiviral nucleosides: 5-alkenyl-1-(2deoxy-2-fluoro-.beta.-D-arabinofuranosyl)uracils. Journal of Medicinal Chemistry, 1984, 27(1), 91-94.

[15] Hertel, L., Kroin, J., Misner, J. and Tustin, J. Synthesis of 2-deoxy-2,2difluoro-D-ribose and 2-deoxy-2,2'-difluoro-D-ribofuranosyl nucleosides. The Journal of Organic Chemistry, 1988, 53(11), 24062409.

[16] Shortnacy-Fowler, A., Tiwari, K., Montgomery, J., Buckheit, Jr., R., Secrist III, J. and Seela, F. Synthesis and Biological Activity of 2'Fluoro-D arabinofuranosyl pyrazolo[3,4-d]pyrimidine Nucleosides. Helvetica Chimica Acta, 1999, 82(12), 2240-2245.

[17] Peng, C. and Damha, M. Polymerase-Directed Synthesis of 2‘-Deoxy2 'fluoro- $\beta$-D-arabinonucleic Acids. Journal of the American Chemical Society, 2007, 129(17), 5310-5311.

[18] Peng, C. and Damha, M. G-quadruplex induced stabilization by 2'deoxy-2'-fluoro-d-arabinonucleic acids (2'F-ANA). Nucleic Acids Research, 2007, 35(15), 4977-4988.

[19] Bakhatoma, D. and Sayed, H. Synthesis, characterization and antioxidant evaluation of novel spiro-5-(fluoren-9-yl)-6-azauracil and their N,N-dialkyl derivatives. 2020.

[20] Bakhotmah, D. and Abdel-Rahman, R. A Review on the Synthesis and Chemistry of Bioactive Pyrazolines Bearing 1,2,4-Triazine Moieties. Mini-Reviews in Organic Chemistry, 2016, 13(1), pp.62-77.

[21] Abdel-Rahman, R., Angawi, R. and Al-Mehmadi, A. Synthesis and biological evaluation of fluorine substituted pyrazolo[4,3e] $[1,2,4]$ triazines as purine analogues. Journal of Saudi Chemical Society, 2017, 21(4), 495-503.

[22] Bakhotmah, D. and Abdel-Rahman, R. Synthesis and Structural Determination of Novel Fluorinated Steroidal Spiro(pyrazolo[4,3e][1,2,4] triazin-3'-yl) Derivatives as Affecting Enzymatic Agents. Letters in Organic Chemistry, 2017, 14(2), pp.134-140.

[23] Abdel-Rahman, R. Reaction of 3-hydazino-5,6-diphenyl-1,2,4-triazine with unsymmetrical 1,3-bicarbonyl compounds. Indian journal of heterocyclic chemistry, 1988, 27(13), 548-553.

[24] Shestakova, T., Deev, S., Ulomsky, E., Rusinov, V., Chupakhin, O., D’yachenko, O., Kazheva, O., Chekhlov, A., Slepukhin, P. and Kodess, M. New approach to the synthesis of azauracils and azaisocytosines. Russian Chemical Bulletin, 2006, 55(11), 2071-2080.

[25] Wahlqvist and Mark, L. Antioxidant relevance to human health. Asia Pacific Journal of Clinical Nutrition, 2013, 22(2), 171-176.

[26] Brewer, M., Natural Antioxidants: Sources, Compounds, Mechanisms of Action, and Potential Applications. Comprehensive Reviews in Food Science and Food Safety, 2011, 10(4), 221-247.

[27] Siddhuraju, P. and Becker, K.The antioxidant and free radical scavenging activities of processed cowpea (Vigna unguiculata (L.) Walp.) seed extracts. Food Chemistry, 2007, 101(1), 10-19. 